\title{
Электрон-фононное взаимодействие в квантовых ямах на основе одноосных материалов
}

\author{
(C) А.Ю. Маслов, О.В. Прошина ฯ \\ Физико-технический институт им. А.Ф. Иоффре Российской академии наук, \\ 194021 Санкт-Петербург, Россия \\ ฯ E-mail: proshina.ton@mail.ioffe.ru
}

Поступила в Редакцию 25 июня 2019 г.

В окончательной редакции 23 июля 2019 г.

Принята к публикации 25 июля 2019 г.

\begin{abstract}
Теоретически исследовано взаимодействие заряженных частиц с интерфейсными оптическими фононами в квантовых ямах, изготовленных с использованием одноосных материалов. Показано, что характер такого взаимодействия существенно зависит от степени анизотропии фононного спектра материалов квантовой ямы и барьеров. При сильной анизотропии взаимодействие оказывается значительно меньше, чем в аналогичных структурах, изготовленных из материалов кубической симметрии. В случае слабой фононной анизотропии найдены условия, при которых взаимодействие заряженных частиц с оптическими фононами можно описывать константой связи фрелиховского типа. При этом в различных структурах может реализоваться как слабое, так и сильное электрон-фононное взаимодействие. Полученные результаты расширяют возможности оптической диагностики квантовых наноструктур.
\end{abstract}

Ключевые слова: квантовая яма, одноосные материалы, интерфейсные оптические фононы, электронфононное взаимодействие, полярон.

DOI: 10.21883/FTP.2019.12.48618.9198

\section{1. Введение}

В наноструктурах взаимодействие заряженных частиц с оптическими фононами оказывается значительно более сложным, чем в объемных материалах $[1,2]$. Это связано с тем, что спектр элементарных возбуждений в таких структурах содержит новые ветви, возникновение которых обусловлено эффектами размерного квантования. В общем случае интенсивность электрон-фононного взаимодействия нельзя описать с помощью некой единой константы связи.

В узких квантовых ямах, однако, взаимодействие заряженных частиц с оптическими фононами определяется главным образом интерфейсными фононами. При этом как при сильном [3], так и при слабом [4] взаимодействии можно ввести эффективную константу связи. Эта величина определяется эффективной массой носителей в квантовой яме и диэлектрическими свойствами барьеров и не сводится к постоянной Фрелиха для какого-либо материала.

В работах $[3,4]$ предполагалось, что и квантовая яма, и барьеры изготовлены из материалов с кубической симметрией. Многие структуры на основе соединений $\mathrm{A}^{\mathrm{II}} \mathrm{B}^{\mathrm{VI}}$ имеют более низкую симметрию, например гексагональную. При этом может значительно измениться характер фононного спектра. Поэтому результаты, полученные в рамках „кубического“ приближения, оказываются неприменимы. Так, в работе [5] использование „кубического“ приближения для квантовой ямы $\mathrm{ZnO} / \mathrm{ZnMgO}$ привело к значительному расхождению теоретических оценок с данными эксперимента.
В настоящей работе найден спектр интерфейсных фононов и построена теория электрон-фононного взаимодействия в квантовых ямах, обладающих одноосной симметрией. Это означает, что оптическая ось гексагонального материала направлена перпендикулярно плоскости квантовой ямы. Подобные наноструктуры в настоящее время уже исследуются экспериментально [6,7]. Именно в этом случае оказывается возможным получить достаточно простые аналитические выражения для параметров электрон-фононного взаимодействия. При другой ориентации оптической оси параметры взаимодействия достаточно сложным образом зависят от ориентации оптических осей материалов барьеров и квантовой ямы. Условия сильного электрон-фононного взаимодействия при этом не реализуются.

\section{2. Спектр интерфейсных фононов}

Найдем спектр интерфейсных оптических фононов, следуя процедуре, предложенной Мори и Андо [8]. В области фононных частот диэлектрическая проницаемость кристалла может быть представлена в стандартном виде

$$
\varepsilon(0)=\varepsilon_{\infty} \frac{\omega^{2}-\omega_{L O}^{2}}{\omega^{2}-\omega_{T O}^{2}},
$$

где $\varepsilon_{\infty}-$ высокочастотный вклад в диэлектрическую проницаемость, $\omega_{L O}$ и $\omega_{T O}-$ частоты продольных и поперечный оптических фононов соответственно. Для одноосных материалов в выбранной геометрии тензор диэлектрической проницаемости имеет диагональный 
вид

$$
\varepsilon(\omega)=\left(\begin{array}{ccc}
\varepsilon_{x x}(\omega) & 0 & 0 \\
0 & \varepsilon_{y y}(\omega) & 0 \\
0 & 0 & \varepsilon_{z z}(\omega)
\end{array}\right) .
$$

При этом плоскость хоу совпадает с плоскостью ямы и $\varepsilon_{x x}(\omega)=\varepsilon_{y y}(\omega)=\varepsilon_{\perp}(\omega)$. Направление оси $z$ совпадает с оптической осью и для него $\varepsilon_{z z}(\omega)=\varepsilon_{\|}(\omega) \neq \varepsilon_{\perp}(\omega)$.

В дальнейшем все величины, относящиеся к материалу квантовой ямы, будем отмечать индексом $(w)$, а к материалу барьеров - индексом $(b)$. В случае, когда и квантовая яма, и барьеры, которые считаются симметричными, изготовлены из одноосных материалов, то, следуя [8], получим уравнения для спектра интерфейсных фононов в следующем виде:

$$
\varepsilon_{\|}^{(b)}(\omega) \gamma^{(b)}(\omega)=-\varepsilon_{\|}^{(w)}(\omega) \gamma^{(w)}(\omega) \operatorname{th}\left(\frac{q a}{2}\right) \gamma^{(w)}(\omega)
$$

для симметричной моды и

$$
\varepsilon_{\|}^{(b)}(\omega) \gamma^{(b)}(\omega)=-\varepsilon_{\|}^{(w)}(\omega) \gamma^{(w)}(\omega) \operatorname{cth}\left(\frac{q a}{2} \gamma^{(w)}(\omega)\right)
$$

для антисимметричной моды. Здесь

$$
\gamma^{(b)}(\omega)=\sqrt{\frac{\varepsilon_{\perp}^{(b)}(\omega)}{\varepsilon_{\|}^{(b)}(\omega)}} \quad \text { и } \quad \gamma^{(w)}(\omega)=\sqrt{\frac{\varepsilon_{\perp}^{(w)}(\omega)}{\varepsilon_{\|}^{(w)}(\omega)}}
$$

- безразмерные параметры, которые меняются от нуля до бесконечности при изменении частоты в интервалах от $\omega_{L O \|}$ до $\omega_{L O \perp}$ и от $\omega_{T O \|}$ до $\omega_{T O \perp}$. Уравнения (3) и (4) имеют решения в областях частот, где $\varepsilon_{\perp}^{(b)}(\omega)$ и $\varepsilon_{\perp}^{(w)}(\omega)$ имеют разные знаки, а пары величин $\varepsilon_{\perp}^{(b)}(\omega)$ и $\varepsilon_{\|}^{(b)}(\omega), \varepsilon_{\perp}^{(w)}$ и $\varepsilon_{\|}^{(w)}$ имеют одинаковые знаки. В общем случае имеется по два решения для каждого из уравнений (3) и (4), удовлетворяющих этим условиям. Для определенности будем считать, что и в яме, и в барьерах выполняются следующие соотношения:

$$
\omega_{L O \perp}>\omega_{L O \|}, \quad \omega_{T O \perp}>\omega_{T O \|} \cdot
$$

Кроме того, будем считать, что фононные частоты материалов квантовой ямы и барьеров не перекрываются. В этом случае уравнения (3) и (4) имеют следующие пары решений. Первая из них оказывается в области фононных частот барьеров. При $q a \rightarrow 0$ получим

$$
\omega_{s 0}^{(1)} \approx \omega_{L O \|}^{(b)}, \quad \omega_{a}^{(1)} \approx \omega_{T O \perp}^{(b)} .
$$

В области $q a \rightarrow \infty$ решения уравнений (3) и (4) совпадают и лежат в интервале

$$
\omega_{T O \perp}^{(b)}<\omega_{s \infty}^{(1)}<\omega_{L O \|}^{(b)} .
$$

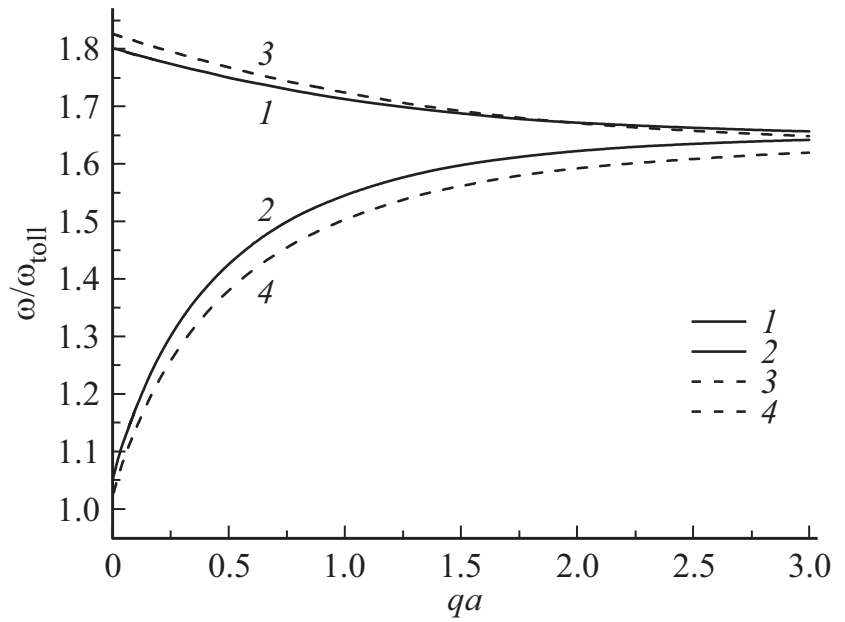

Рис. 1. Спектр интерфейсных фононов в случае слабой фононной анизотропии. 1 - симметричная, 2 - антисимметричная мода для одноосного материала, 3 - симметричная, 4 - антисимметричная мода для кубического материала.

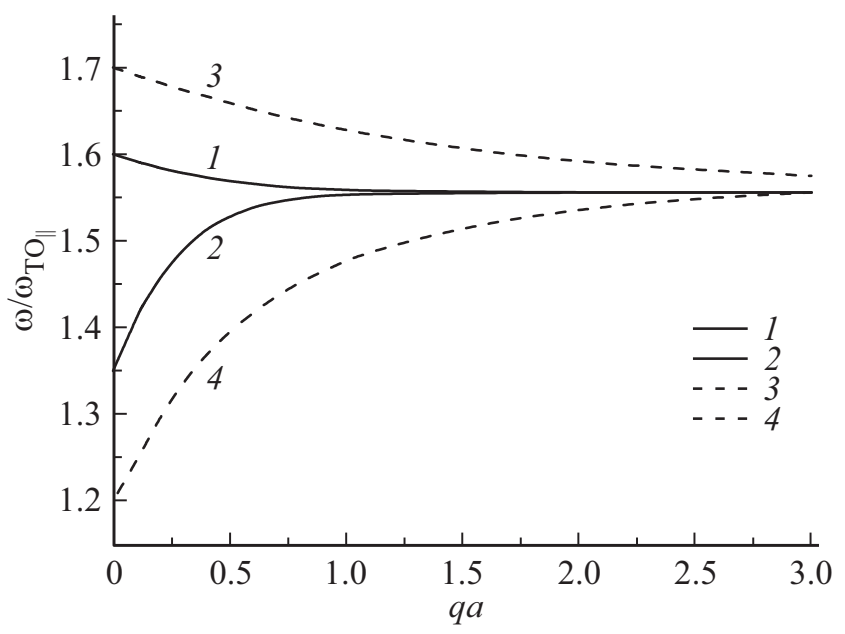

Рис. 2. Спектр интерфейсных фононов в случае сильной фононной анизотропии. 1 - симметричная, 2 - антисимметричная мода для одноосного материала, 3 - симметричная, 4 - антисимметричная мода для кубического материала.

Аналогично вторая пара решений оказывается в области фононных частот материала квантовой ямы. При $q a \rightarrow 0$ получим

$$
\omega_{s 0}^{(2)} \approx \omega_{L O \|}^{(w)}, \quad \omega_{a}^{(2)} \approx \omega_{T O \perp}^{(w)},
$$

а при $q a \rightarrow \infty$ решения уравнений (3) и (4) совпадают и лежат в интервале

$$
\omega_{T O \perp}^{(w)}<\omega_{s \infty}^{(2)}<\omega_{L O \|}^{(w)}
$$

В том случае, когда яма (или барьеры) изготовлены из материалов кубической симметрии, выражения (3) и (4) несколько упрощаются. В этих случаях надо подставить $\gamma^{(i)}(\omega)=1$, где $i=b, w$ для материала барьеров и ямы соответственно. Спектр интерфейсных фононов 
представлен на рис. 1 для случая слабой фононной анизотропии. Здесь использованы следующие модельные соотношения частот $\omega$ :

$$
\begin{aligned}
& \omega_{T O \perp}=1.05 \omega_{T O \|}, \\
& \omega_{L O \perp}=1.8 \omega_{T O \|}, \\
& \omega_{L O \perp}=1.85 \omega_{T O \|} .
\end{aligned}
$$

Для случая сильной фононной анизотропии спектр интерфейсных фононов приведен на рис. 2 при соотношении частот:

$$
\begin{aligned}
& \omega_{T O \perp}=1.35 \omega_{T O \|}, \\
& \omega_{L O \|}=1.6 \omega_{T O \|}, \\
& \omega_{L O \perp}=1.8 \omega_{T O \|} .
\end{aligned}
$$

Для сравнения на рис. 1 и 2 пунктирными линиями представлен спектр в „кубическом“ приближении $\gamma^{(b)}(\omega)=\gamma^{(w)}(\omega)=1$. При этом для „кубического“ приближения были использованы средние арифметические значения частот $\omega_{L O \perp}$ и $\omega_{L O \|}$, а также $\omega_{T O \perp}$ и $\omega_{T O \|}$ для материалов квантовой ямы и барьеров.

Отметим, что области возможных значений частот интерфейсных фононов оказались несколько уже, чем в случае гетероструктур на основе кубических материалов. Использование „кубического“ приближения при любом способе усреднения фононных частот дает не вполне точные результаты даже для спектра интерфейсных фононов. Эти расхождения увеличиваются с ростом степени фононной анизотропии использованных материалов. Таким образом, изучение спектра интерфейсных фононов позволяет независимым образом определять диэлектрические параметры объемных материалов, использованных при изготовлении квантовой ямы.

Различия между квантовыми ямами на основе кубических и гексагональных материалов оказываются более значительными при исследовании электрон-фононного взаимодействия.

\section{3. Особенности электрон-фононного взаимодействия}

Выражение для электрон-фононного взаимодействия получим по схеме, предложенной в [8], с учетом более низкой симметрии диэлектрической проницаемости материалов ямы и барьеров. Его можно представить в следующем виде:

$$
\begin{aligned}
\hat{H}_{e-\mathrm{ph}, s \pm}= & \sum_{\mathbf{q}}\left(\frac{2 \pi \omega_{s \pm} e^{2}}{2 L^{2}}\right)^{1 / 2} \frac{\exp [i \mathbf{q} \rho]}{\sqrt{2 q}} f_{s}(\mathbf{q}, z) \\
& \times F_{s \pm}\left(\mathbf{q}, \omega_{s \pm}\right)\left(\hat{a}_{s \pm}(\mathbf{q})+\hat{a}_{s \pm}^{+}(\mathbf{q})\right)
\end{aligned}
$$

для симметричных мод $s \pm$ и аналогично для антисимметричных мод $a \pm$ :

$$
\begin{aligned}
\hat{H}_{e-\mathrm{ph}, a \pm}= & \sum_{\mathbf{q}}\left(\frac{2 \pi \omega_{a \pm} e^{2}}{2 L^{2}}\right)^{1 / 2} \frac{\exp [i \mathbf{q} \rho]}{\sqrt{2 q}} f_{a}(\mathbf{q}, z) \\
& \times F_{a \pm}\left(\mathbf{q}, \omega_{a \pm}\right)\left(\hat{a}_{a \pm}(\mathbf{q})+\hat{a}_{a \pm}^{+}(\mathbf{q})\right) .
\end{aligned}
$$

В выражениях (12) и (13) $L^{2}-$ нормировочная площадь поверхности квантовой ямы, q - двумерный вектор фонона в плоскости ямы, $\hat{a}_{ \pm}(\mathbf{q})$ и $\hat{a}_{ \pm}^{+}(\mathbf{q})-$ операторы уничтожения и рождения соответствующих оптических фононов. Множители $f_{s}(\mathbf{q}, z)$ и $f_{a}(\mathbf{q}, z)$ отражают распределение интенсивности симметричного и антисимметричного возбуждений по направлению, перпендикулярному плоскости квантовой ямы. Они имеют вид

$$
f_{s}(\mathbf{q}, z)=\left\{\begin{array}{l}
\exp \left[i \gamma^{(b)}(\omega) q(z+a / 2)\right] ; \quad z \leq-\frac{a}{2}, \\
\frac{\operatorname{ch}\left[q \gamma^{(w)}(\omega) z\right]}{\operatorname{ch}\left[q \gamma^{(w)}(\omega) a / 2\right]} ; \quad-\frac{a}{2} \leq z \leq \frac{a}{2}, \\
\exp \left[-i \gamma^{(b)}(\omega) q(z-a / 2)\right] ; \quad z \geq \frac{a}{2}
\end{array}\right.
$$

для симметричной моды и

$$
f_{s}(\mathbf{q}, z)=\left\{\begin{array}{l}
\exp \left[\gamma^{(b)}(\omega) q(z+a / 2)\right] ; \quad z \leq-\frac{a}{2}, \\
\frac{\operatorname{sh}\left[q \gamma^{(w)}(\omega) z\right]}{\operatorname{sh}\left[q \gamma^{(w)}(\omega) a / 2\right]} ; \quad-\frac{a}{2} \leq z \leq \frac{a}{2} \\
\exp \left[\gamma^{(b)}(\omega) q(z-a / 2)\right] ; \quad z \geq \frac{a}{2}
\end{array}\right.
$$

для антисимметричной моды. Множители $\gamma^{(w)}(\omega)$ и $\gamma^{(b)}(\omega)$ изменяются от нуля до бесконечности, как правило, при небольшом изменении частоты (от $\omega_{L O \|}^{(b)}$ до $\omega_{L O \perp}^{(b)}$ и в других аналогичных частотных интервалах). Поэтому частотная зависимость для параметров электрон-фононного взаимодействия оказывается очень сильной. Основной вклад в эту зависимость определяется значениями множителей $F_{s \pm}\left(\mathbf{q}, \omega_{s \pm}\right)$ и $F_{a \pm}\left(\mathbf{q}, \omega_{s \pm}\right)$, которые имеют вид

$$
\begin{aligned}
F_{s \pm}\left(\mathbf{q}, \omega_{s \pm}\right)= & \frac{1}{2}\left\{\left(\gamma^{(w)}\left(\beta_{\perp}^{(w)}\right)^{-1}+\frac{\left(\beta_{\|}^{(w)}\right)^{-1}}{\gamma^{(w)}}\right) \operatorname{th}\left[\gamma^{(w)} \mathbf{q} \frac{a}{2}\right]\right. \\
& \left.+\frac{1}{2}\left(\gamma^{(b)}\left(\beta_{\perp}^{(b)}\right)^{-1}+\frac{\left(\beta_{\|}^{(b)}\right)^{-1}}{\gamma^{(b)}}\right)\right\}^{-1 / 2}, \\
F_{a \pm}\left(\mathbf{q}, \omega_{s \pm}\right)= & \frac{1}{2}\left\{\left(\gamma^{(w)}\left(\beta_{\perp}^{(w)}\right)^{-1}+\frac{\left(\beta_{\|}^{(w)}\right)^{-1}}{\gamma^{(w)}}\right) \operatorname{cth}\left[\gamma^{(w)} \mathbf{q} \frac{a}{2}\right]\right. \\
& \left.+\frac{1}{2}\left(\gamma^{(b)}\left(\beta_{\perp}^{(b)}\right)^{-1}+\frac{\left(\beta_{\|}^{(b)}\right)^{-1}}{\gamma^{(b)}}\right)\right\}^{-1 / 2} .
\end{aligned}
$$


Здесь все множители $\beta$ имеют одинаковую частотную зависимость с различными значениями фононных частот. Для примера,

$$
\begin{aligned}
\beta_{\perp}^{(w)} & =\beta_{\perp}^{(w)}(\omega) \\
& =\left(\frac{1}{\varepsilon_{\infty \perp}^{(w)}}-\frac{1}{\varepsilon_{0 \perp}^{(w)}}\right) \frac{\left(\omega_{L O \perp}^{(w)}\right)^{2}}{\omega^{2}}\left(\frac{\omega^{2}-\omega_{T O \perp}^{(w)}}{\omega_{L O \perp}^{(w)}-\omega_{T O \perp}^{(w)}}\right)^{2} .
\end{aligned}
$$

Остальные параметры $\beta$ получаются из (18) заменой соответствующих индексов. Полученные выражения (16), (17) при отсутствии анизотропии переходят в известные выражения для электрон-фононного взаимодействия в квантовых ямах на основе материалов кубической симметрии [8]. Для такого предельного перехода следует положить в $(16),(17) \gamma^{(i)}(\omega)=1$ и $\beta_{\perp}^{(i)}=\beta_{\|}^{(i)}$, где $i=b, w$ для материалов барьеров и ямы соответственно. Однако наличие даже малой анизотропии приводит к качественному изменению характера электронфононного взаимодействия. При этом наиболее существенное влияние связано с анизотропией материала барьеров. Из анализа уравнений (16), (17) с учетом спектра интерфейсных фононов (3), (4) следует, что в области малых значений вектора q параметры $F_{s \pm}\left(\mathbf{q}, \omega_{s \pm}\right)$ и $F_{a \pm}\left(\mathbf{q}, \omega_{s \pm}\right)$ стремятся к нулю. Это означает, что дальнодействующее электрон-фононное взаимодействие, характерное для ионных кристаллов, в рассматриваемых структурах оказывается сильно подавленным.

В зависимости от соотношения параметров, определяющих диэлектрическую анизотропию барьеров, можно выделить две качественно различные ситуации. Для оценки меры анизотропии удобно взять параметр $\Delta$, равный

$$
\Delta=\frac{\left|\omega_{L O \|}^{2}-\omega_{L O \perp}^{2}\right|}{\omega_{L O \|}^{2}} .
$$

Если параметр $\Delta$ достаточно велик (порядка единицы), то взаимодействие заряженных частиц с интерфейсными оптическими фононами оказывается слабым. В соответствии с рис. 2 спектр интерфейсных фононов сильно отличается от результатов, полученных в „кубическом“ приближении. Электрон-фононное взаимодействие при этом можно учитывать по теории возмущений. Образование связанного состояния заряженных частиц и фононов - поляронов большого радиуса — в этом случае не происходит. Для корректного учета электрон-фононного взаимодействия, помимо интерфейсных фононов, необходимо учитывать также и другие типы возбуждений, а именно фононы, локализованные в квантовой яме [9] и необыкновенные фононы [10]. Аналитическое описание электрон-фононного взаимодействия, даже в рамках теории возмущений, оказывается очень громоздким.

Другая ситуация возникает при слабой анизотропии фононного спектра, когда параметр $\Delta$ из выражения (19) оказывается малым $(\Delta \ll 1)$. Для большинства соединений $\mathrm{A}^{\mathrm{II}} \mathrm{B}^{\mathrm{VI}}$ реализуется именно такое значение параметра $\Delta$.
В этом случае существует интервал значений волнового вектора фонона q, в котором

$$
\Delta \ll q a<1 .
$$

В этом интервале взаимодействие заряженных частиц с интерфейсными оптическими фононами оказывается параметрически велико по сравнению с взаимодействием с другими ветвями фононного спектра. При выполнении условия (20) основной вклад в электрон-фононное взаимодействие сводится к двумерному взаимодействию фрелиховского типа, с константой взаимодействия

$$
\alpha_{\mathrm{eff}}=\frac{e^{2}}{2 \hbar \omega^{(b)}}\left(\frac{2 m^{(w)} \omega^{(b)}}{\hbar}\right)^{1 / 2} \frac{1}{\varepsilon_{\mathrm{opt}}^{(b)}} .
$$

Здесь

$$
\frac{1}{\varepsilon_{\mathrm{opt}}^{(b)}}=\frac{1}{\varepsilon_{\infty}^{(b)}}-\frac{1}{\varepsilon_{0}^{(b)}}
$$

- обратная оптическая диэлектрическая проницаемость. В данном приближении различием между продольными и поперечными параметрами барьеров можно пренебречь. Отметим, что эффективная константа взаимодействия $\alpha_{\text {eff }}$ зависит как от параметров материала квантовой ямы, так и от параметров барьеров. Поэтому она оказывается характеристикой структуры, а не какого-либо одного конкретного материала. Основное отличие данных структур от тех, которые изготовлены с использованием материалов кубической симметрии, заключается в том, что область существования взаимодействия фрелиховского типа ограничена по значениям волнового вектора не только сверху, но и снизу.

В зависимости от величины параметра $\alpha$ eff в структуре возможно как сильное (при $\alpha_{\text {eff }}>1$ ), так и слабое (при $\left.\alpha_{\text {eff }}<1\right)$ электрон-фононное взаимодействие. В случае сильного взаимодействия в квантовой яме должны возникать поляроны большого радиуса, представляющие собой связанное состояние заряженной частицы и интерфейсных оптических фононов. Радиус поляронного состояния $r_{\text {pol }}$ оказывается равным:

$$
r_{\mathrm{pol}}=\frac{\hbar^{2}\left(\varepsilon_{\mathrm{opt}}^{(b)}\right)^{2}}{m^{(w)} e^{2}} .
$$

При этом такое рассмотрение будет самосогласованным только в том случае, когда радиус поляронного состояния удовлетворяет соотношению

$$
\Delta \ll \frac{a}{r_{\text {pol }}}<1
$$

которое обусловлено условием (20). Если радиус поляронного состояния (22) не удовлетворяет условию (23), то возникновение поляронных состояний оказывается невозможным. 


\section{4. Заключение}

В настоящей работе получены аналитические выражения для спектра интерфейсных фононов и параметров электрон-фононного взаимодействия в квантовых ямах, изготовленных из материалов одноосной симметрии. Показано, что в структурах с квантовыми ямами при использовании материалов одноосной симметрии, взаимодействие заряженных частиц с полярными оптическими фононами оказывается, как правило, меньше, чем при использовании материалов кубической симметрии. Тем не менее при выполнении условия (23) возможно формирование поляронов большого радиуса. При нарушении неравенства (23) взаимодействие заряженных частиц с оптическими фононами можно описывать по теории возмущений. При этом необходимо учитывать все ветви оптических фононов, возможные в данной структуре. Исследование подобных возбуждений дает фундаментально новую информацию об оптических и транспортных свойствах квантовых ям. Это расширяет возможности диагностики наноструктур неразрушающими оптическими методами. Полученные результаты также показывают, что изучение спектра интерфейсных оптических фононов позволяет независимым образом определять параметры объемных материалов, использованных при изготовлении наноструктуры.

\section{Конфликт интересов}

Авторы заявляют, что у них нет конфликта интересов.

\section{Список литературы}

[1] Z. Wang, K. Reinhardt, M. Dutta, M.A. Stroscio. Phonons in bulk and low-dimensional systems. In: Length-Scale Dependent Phonon Interactions ed. by S.L Shinde, G.P. Srivastava (Springer, 2014).

[2] B.K. Ridley. Hybrid Phonons in Nanostructures (Oxford University Press, UK, 2017).

[3] А.Ю. Маслов, О.В. Прошина. ФТП, 44 (2), 200 (2010).

[4] А.Ю. Маслов, О.В. Прошина. ФТП, 49 (10), 1389 (2015).

[5] Y. Imanaka, T. Takamasu, H. Tampo, H. Shibata, S. Niki. Phys. Status Solidi C, 7, 1599 (2010).

[6] P.I. Kuznetsova, V.A. Jitova, G.G. Yakushchevaa, B.S. Shchamkhalova, L.Yu. Zakharova, V.I. Kozlovskyb, Ya.K. Skasyrskyb, K.P. O’Donnellc, C. Trager-Cowan. Physica E, 17, 516 (2003).

[7] M. Lopez-Ponce, A. Nakamura, M. Suzuki, J. Temmyo, S. Agouram, M.C. Martínez-Tomás, V. Muñoz-Sanjosé, P. Lefebvre, J.M. Ulloa, E. Muñoz, A. Hierro. Nanotechnology, 25, 255202 (2014).

[8] M. Mori, T. Ando. Phys. Rev. B, 40, 6175 (1989).

[9] Z. Wang, K. Reinhardt, M. Dutta, M.A. Stroscio. In: LengthScale Dependent Phonon Interactions, ed. by S.L. Shindé, G.P. Srivastava (Springer Science + Business Media, N.Y., USA, 2014) Chap. 2.

[10] A.Yu. Maslov, O.V. Proshina. In: Phonons in Low Dimensional Structures, ed. by V.N. Stavrou (IntechOpen Limited, London, UK, 2018) Chap. 1, p. 3.

Редактор Г.А. Оганесян

\section{Electron-phonon interaction in quantum wells based on uniaxial materials}

\author{
A.Yu. Maslov, O.V. Proshina \\ loffe Institute, \\ 194021 St. Petersburg, Russia
}

\begin{abstract}
The interaction of charged particles with interface optical phonons is studied theoretically in quantum wells based on uniaxial symmetry materials. It is shown that this interaction depends on the phonon spectrum anisotropy. With strong anisotropy degree, the interaction turns out to be significantly less than one in similar structures made of cubic symmetry materials. In the case of weak phonon anisotropy, conditions are found under which the interaction of charged particles with optical phonons can be described by the coupling constant of the Fröhlich type. It turns out that both weak and strong electron-phonon interaction can be realized in various structures. The obtained results expand the possibilities of optical diagnostics of quantum nanostructures.
\end{abstract}

